\title{
The Electronic and Magnetic Properties of the $\mathrm{USn}_{2}$ Compound
}

\author{
A. SZAJEK \\ Institute of Molecular Physics, Polish Academy of Sciences \\ Smoluchowskiego 17, 60-179 Poznań, Poland \\ (Received August 27, 2003; revised version April 20, 2004) \\ The $a b$ initio self-consistent calculations were performed for $\mathrm{USn}_{2}$ com- \\ pound, which crystallizes in the $\mathrm{ZrGa}_{2}$-type structure ( $\mathrm{Cmmm}$ space group). \\ The tight binding linear muffin-tin orbital method in the atomic sphere ap- \\ proximation was used in the calculations. The spin-polarized calculations \\ confirmed the antiferromagnetic order in the $\mathrm{USn}_{2}$ system. The magnetic \\ moment is predominantly located on the uranium atoms and is formed \\ mainly by the $f$ electrons. The three types of Sn atoms may be treated
} as non-magnetic.

PACS numbers: 71.20.-b, 75.25.+z

\section{Introduction}

Recently uranium compounds have attracted interest because of many interesting properties such as the Pauli paramagnetism, spin fluctuations, heavy fermions, magnetic ordering, or superconductivity. Wide range of properties in these compounds is caused by the uranium $5 f$ electrons, which show an intermediate character between the localized $4 f$ electron system and itinerant character of $3 d$ electrons. The way to understand these properties is systematic studying of the phase diagrams of the binary phases, where the same atoms located in different crystallographic lattices lead to unique phenomena.

The first reference on the binary U-Sn was by Treick et al. [1]. The existence of three binary compounds was reported: $\mathrm{USn}_{3}, \mathrm{U}_{3} \mathrm{Sn}_{5}$, and $\mathrm{U}_{3} \mathrm{Sn}_{2}$. This paper was a basis for a tentative phase diagram proposed by Sheldon et al. [2]. These investigations led to conclusion that compounds such as $\mathrm{U}_{3} \mathrm{Sn}_{7}, \mathrm{USn}_{2}, \mathrm{U}_{5} \mathrm{Sn}_{4}$, and USn reported by Sari et al. [3] are not truly equilibrium phases. The comprehensive 
work by Palenzona and Manfrinetti [4], in which the techniques of differential thermal analysis, metallographic analysis, X-ray diffraction, and electron microscopy were employed, has removed the uncertainty in this matter. The following intermediate, homogeneous phases have been confirmed: $\mathrm{U}_{5} \mathrm{Sn}_{4}, \mathrm{USn}, \mathrm{USn}_{2}, \mathrm{U}_{3} \mathrm{Sn}_{7}$, and $\mathrm{USn}_{3}$. The $\mathrm{U}_{3} \mathrm{Sn}_{5}$ and $\mathrm{U}_{3} \mathrm{Sn}_{2}$ systems were found to be non-homogeneous. The latest reinvestigation of the structural chemistry of the binary stannides [5] confirmed the following phases reported earlier [4]: $\mathrm{USn}_{3}$ ( $\mathrm{AuCu}_{3}$-type), $\mathrm{U}_{3} \mathrm{Sn}_{7}$ ( $\mathrm{Ce}_{3} \mathrm{Sn}_{7}$-type), $\mathrm{USn}_{2}$ (ZrGa ${ }_{2}$-type), USn (ThIn-type), and $\mathbf{U}_{5} \mathrm{Sn}_{4}\left(\mathrm{Ti}_{5} \mathrm{Ga}_{4}\right.$-type).

The magnetic measurements $[5,6]$ showed that $\mathbf{U}_{3} \mathrm{Sn}_{7}$ and $\mathrm{USn}_{2}$ are antiferromagnets, USn and $\mathrm{U}_{5} \mathrm{Sn}_{4}$ are ferromagnets and $\mathrm{USn}_{3}$ is a spin fluctuating system. The temperatures of magnetic ordering are in the range between $49 \mathrm{~K}$ and $80 \mathrm{~K}[5,6]$. A comparison of this behaviour to that of the isostructural binary germanides reveals an interesting evolution of magnetic interactions connected to the hybridization of $\mathrm{U}(5 f)$ electrons with those of the ligands (Sn, Ge). The antiferromagnetic order was confirmed for $\mathbf{U}_{3} \mathrm{Sn}_{7}$ and $\mathrm{USn}_{2}$ by neutron diffraction [7].

As a first step toward an understanding of the electronic states in the whole temperature range, it is important to clarify the ground state properties of these compounds. Recently developed theoretical methods to carrying out reliable qualitative calculations of energy band structures are very powerful tools. However, it is a big challenge to calculate band structure of $5 f$ electron systems. The degree of localization of the $\mathrm{U}(5 f)$ states is not known and analysis of interuranium distances indicates possible different degree of localization of $5 f$ electrons. Till now, for binary U-Sn systems, the band structure calculations have been reported for two compounds: $\mathrm{USn}_{3}$ [8-11] and USn [12]. The first compound has simple unit cell $\left(\mathrm{AuCu}_{3}\right.$-type) but the problem was to take into account many body effects responsible for large enhancement in the specific heat coefficient, which in the case of $\mathrm{USn}_{3}$ is about seven times larger than for $\mathrm{UGe}_{3}$. The second compound, USn (ThIn-type structure), has much more complex unit cell: uranium and tin atoms each occupy three crystallographic positions marked as $4 \mathrm{~d}, 4 \mathrm{~d}$, and $4 \mathrm{c}$ sites. In this structure the $\mathbf{U}(4 \mathrm{c})$ atoms form metallic chains running along $c$ axis, with interatomic $\mathbf{U}(4 c)-\mathrm{U}(4 \mathrm{c})$ distance of $3.10 \AA$, which is close to the Hill limit, and the remaining interatomic distances between uranium atoms are well above the limit. USn orders ferromagnetically with an ordered moment of $1.2 \mu_{\mathrm{B}} / \mathrm{U}$ [5]. Because of the short $\mathbf{U}(4 c)-\mathbf{U}(4 c)$ distance and expected hybridization between $f$ states and the conduction-band electron states it was interesting to know if this uranium sublattice is also magnetically ordered. In the isostructural germanide UGe situation was similar but in this compound $\mathbf{U}(4 \mathrm{c})-\mathrm{U}(4 \mathrm{c})$ distance is shorter, about $2.9 \AA$, and calculated spin magnetic moment is reduced to about $0.2 \mu_{\mathrm{B}} / \mathrm{U}$ [13]. The rest of $U$ atoms have magnetic moments about nine times larger and they order antiferromagnetically. In the case of USn, the calculations showed that magnetic moments on $\mathrm{U}$ atoms order ferromagnetically, and the spin moments have similar values $\left(2.7-2.9 \mu_{\mathrm{B}} / \mathrm{U}\right.$ atom), even in the case of $\mathrm{U}(4 \mathrm{c})$ atoms. 
The $\mathrm{USn}_{2}$ compound has relatively tin-rich composition and orders antiferromagnetically. Its isostructural equivalent $\mathbf{U G e}_{2}$ orders ferromagnetically at ambient pressure, and $T_{\mathrm{C}}$ decreases under pressure, vanishing at about $1.6 \mathrm{GPa}$ [14]. Around $1 \mathrm{GPa}$, Saxena et al. [15] have found that $\mathrm{UGe}_{2}$ becomes superconducting while remaining ferromagnetic ( $m \approx 1 \mu_{\mathrm{B}} / \mathrm{U}$ atom) providing a novel example of coexistence of the superconductivity and ferromagnetism. The band calculations performed by Shick and Pickett [16] reproduced the magnitude of the observed magnetic moment and the magnetic anisotropy.

The aim of this paper is to calculate the electronic structure of the $\mathrm{USn}_{2}$ compound and compare the obtained results with the experimental ones.

\section{Method of calculations}

The electronic band structure was calculated by the tight-binding linear muffin-tin orbital method (TB-LMTO) in the atomic sphere approximation (ASA) $[17,18]$. The neutron diffraction [7] experimental values of the lattice parameters were assumed for computations. In the crystal structure of $\mathrm{USn}_{2}$ uranium atoms occupy one position $4 \mathrm{j}$ and tin atoms occupy three crystallographic positions marked as $4 \mathrm{i}, 2 \mathrm{a}$, and $2 \mathrm{c}$ sites (see Fig. 1). The unit cell accommodates four formula units, the positions of atoms are given in Table I. In the ASA the volume of the unit cell is divided into the Wigner-Seitz (W-S) atomic spheres of the different radii, which are collected in Table $I$. The average Wigner-Seitz radius is $1.825 \AA$.

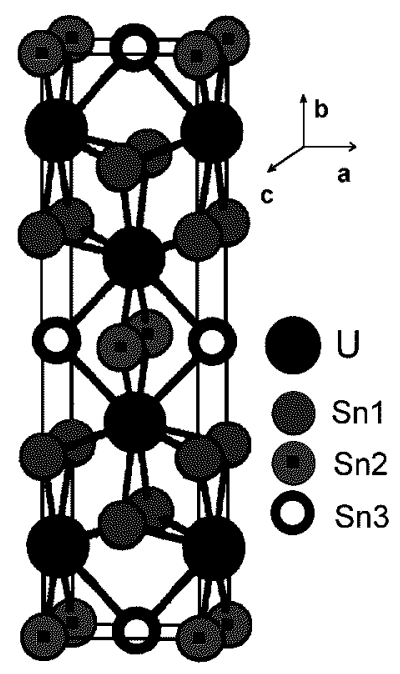

Fig. 1. The crystallographic unit cell of $\mathrm{USn}_{2}$ compound. 


\section{TABLE I}

Structural parameters of $\mathrm{USn}_{2}$ compound (orthorhombic, $\mathrm{ZrGa}_{2}$-type, space group: Cmmm).

\begin{tabular}{c|c|c|c|c}
\hline \hline Atom (site) & $x$ & $y$ & $z$ & $S_{j}[\AA]$ \\
\hline $\mathrm{U}(4 \mathrm{j})$ & 0.0 & $0.1477(4)$ & 0.5 & 1.962 \\
$\operatorname{Sn} 1(4 \mathrm{i})$ & 0.0 & $0.3229(5)$ & 0.0 & 1.742 \\
$\operatorname{Sn} 2(2 \mathrm{a})$ & 0.0 & 0.0 & 0.0 & 1.762 \\
$\mathrm{Sn} 3(2 \mathrm{c})$ & 0.5 & 0.0 & 0.5 & 1.744
\end{tabular}

The starting atomic configurations were assumed as core $+6 p^{6} 5 f^{3} 6 d^{1} 7 s^{2}$ for $\mathrm{U}$, and core $+5 s^{2} 5 p^{2}$ for Sn atoms. The overlap volume of muffin-tin spheres for the chosen $\mathrm{W}-\mathrm{S}$ radii $S_{j}$ was $9.67 \%$. The standard combined corrections for overlapping W-S spheres [17] were applied to compensate the ASA errors. The fully relativistic treatment was used for the core electrons and for the valence electrons the scalar relativistic approximation supplemented by the spin-orbit effects taken into account within the Min and Jang scheme [19] was adopted. The von Barth-Hedin [20] exchange-correlation potential was used. The self-consistent calculations were performed for $1221 k$-points in the irreducible wedge $(1 / 8)$ of the Brillouin zone. The iteration procedure was ended when the self-consistency within the $0.01 \mathrm{mRy}$ error was achieved. For the integration in the $k$-space the standard tetrahedron method was used [21].

\section{Results and discussion}

The spin polarized band structure calculations were performed using the TB LMTO ASA method for double unit cell along $c$ axis, as it was suggested by neutron diffraction (see Fig. 3 in [7]). The uranium atoms in distinct cells were formally treated as different types of atoms, the calculations for them were performed independently up to self-consistency. We also obtained solutions for ferromagnetic order and antiferromagnetic order with antiparallel alignment within single unit cell. In both cases the total energies were higher than for the case described by the neutron diffraction. The presented below results of calculations will concern only this situation.

The calculated electronic structure of $\mathrm{USn}_{2}$ gave density of states (DOS) plots presented in Figs. 2 and 3. The total DOS in Fig. 2A is decomposed into contributions from $s, p, d$, and $f$ electrons in respective panels (let us note the different vertical scales). Site projected DOS plots are presented in Fig. 2B.

In the total DOS a few characteristic features can be observed. The two subbands located between 17.9 and $19.5 \mathrm{eV}$ below the Fermi level (taken as $E_{\mathrm{F}}=0$ ) is due to the $\mathrm{U} p$ electrons $(95.6 \%)$ with small contributions from $\mathrm{Sn}$ atoms ( $s$ and $p$ electrons), which are the nearest neighbours of the $\mathbf{U}$ atoms. The distances between 

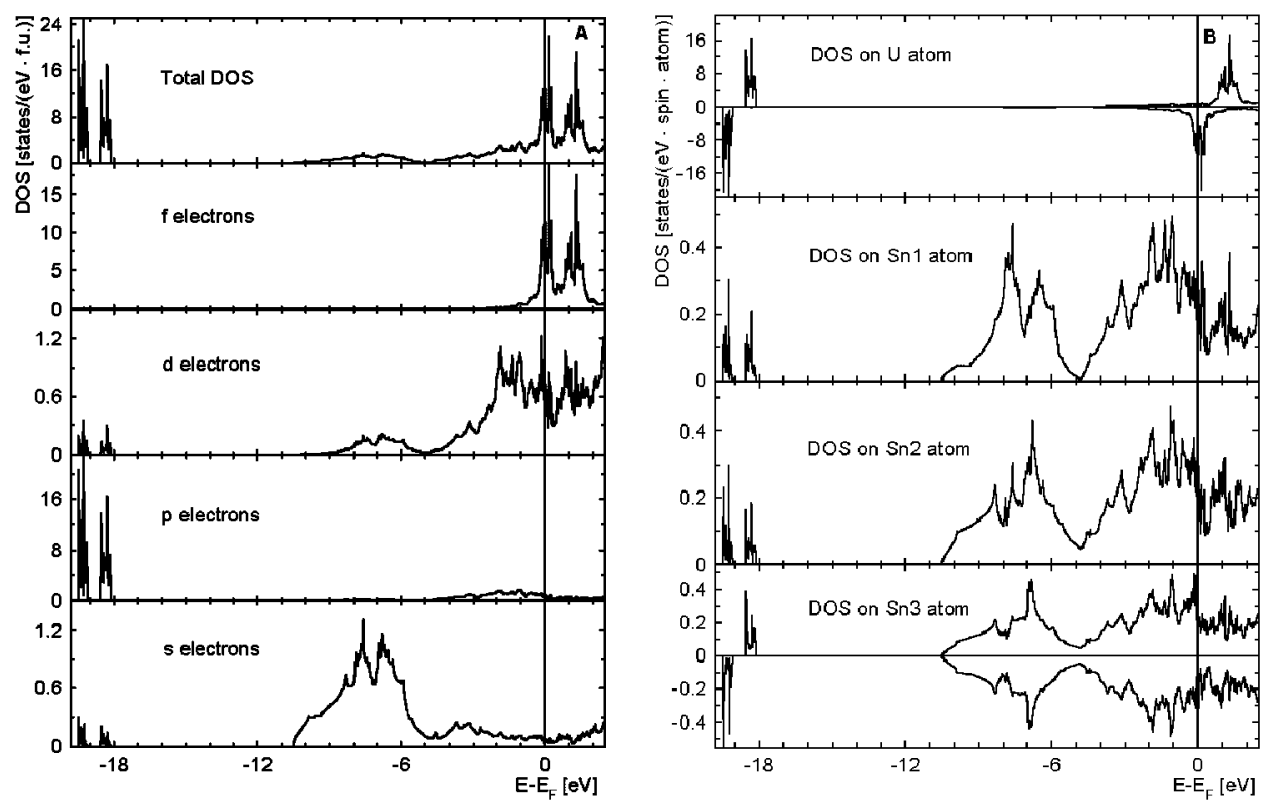

Fig. 2. The DOS plots for $\mathrm{USn}_{2}$ compound: (A) total and l-decomposed; (B) local (let us note that for $\mathrm{U}$ and $\mathrm{Sn} 3$ atoms the DOS plots are presented for two spin directions).

the $\mathrm{U}$ atoms and surrounding atoms are the following: $3.176 \AA, 3.194 \AA, 3.178 \AA$, and $3.858 \AA$ for $\mathrm{Sn} 1, \mathrm{Sn} 2, \mathrm{Sn} 3$, and $\mathrm{U}$ atoms, respectively. The $\mathrm{U}(6 p)$ electrons form subbands, which have about $0.4 \mathrm{eV}$ of width. The similar subbands are formed by $\mathrm{U}(6 p)$ electrons in $\mathrm{UGe}_{2}$ compound [22]. In the case when interuranium distances are larger the $\mathbf{U}(6 p)$ electrons form very narrow bands (e.g. for $\mathrm{UCu}_{5} \mathrm{Sn}$ compound, where $\mathrm{U}-\mathbf{U}$ distance is about $5 \AA[23])$. The range between $-10.5 \mathrm{eV}$ and $-4.8 \mathrm{eV}$ is occupied mainly by $s$ electrons from Sn atoms (see Fig. 3B-D). The DOS located above Sn $s$ electrons up to the Fermi level are composed of $\operatorname{Sn}(p)$ and $U(d, f)$ electrons. The $\mathrm{U}(5 f)$ electrons provide the main contribution to the total DOS at the Fermi level, about $86 \%$ of the value 12.007 states/(eV f.u.). The calculated value of the Sommerfeld coefficient $\gamma_{0}$ in the linear term of the heat capacity is equal to $28.3 \mathrm{~mJ} /\left(\mathrm{mol} \mathrm{K}^{2}\right)$. This value is close to the experimental value $\gamma$ obtained by Dhar et al. [6]: $28 \mathrm{~mJ} /\left(\mathrm{mol} \mathrm{K}^{2}\right)$. The theoretical value should be lower than the experimental one because of an enhancement factor $\lambda, \gamma=(1+\lambda) \gamma_{0}$, which has positive value. Uncertainty of $\gamma_{0}$ is magnified because of location of $E_{\mathrm{F}}$ on the slope of sharp DOS peak. The U(5f) electrons form a relatively wide band occupied by about 3.2 electrons, having the centre of gravity above $E_{\mathrm{F}}$. The occupation numbers and DOS at the Fermi level for particular atoms are collected in Tables II and III, respectively. The occupation numbers collected in Table II allow calculating the spin magnetic moments. The total magnetic moment is predominantly located on $\mathrm{U}$ atoms $\left(2.566 \mu_{\mathrm{B}} / \mathrm{U}\right.$ atom $)$. The main contribution 

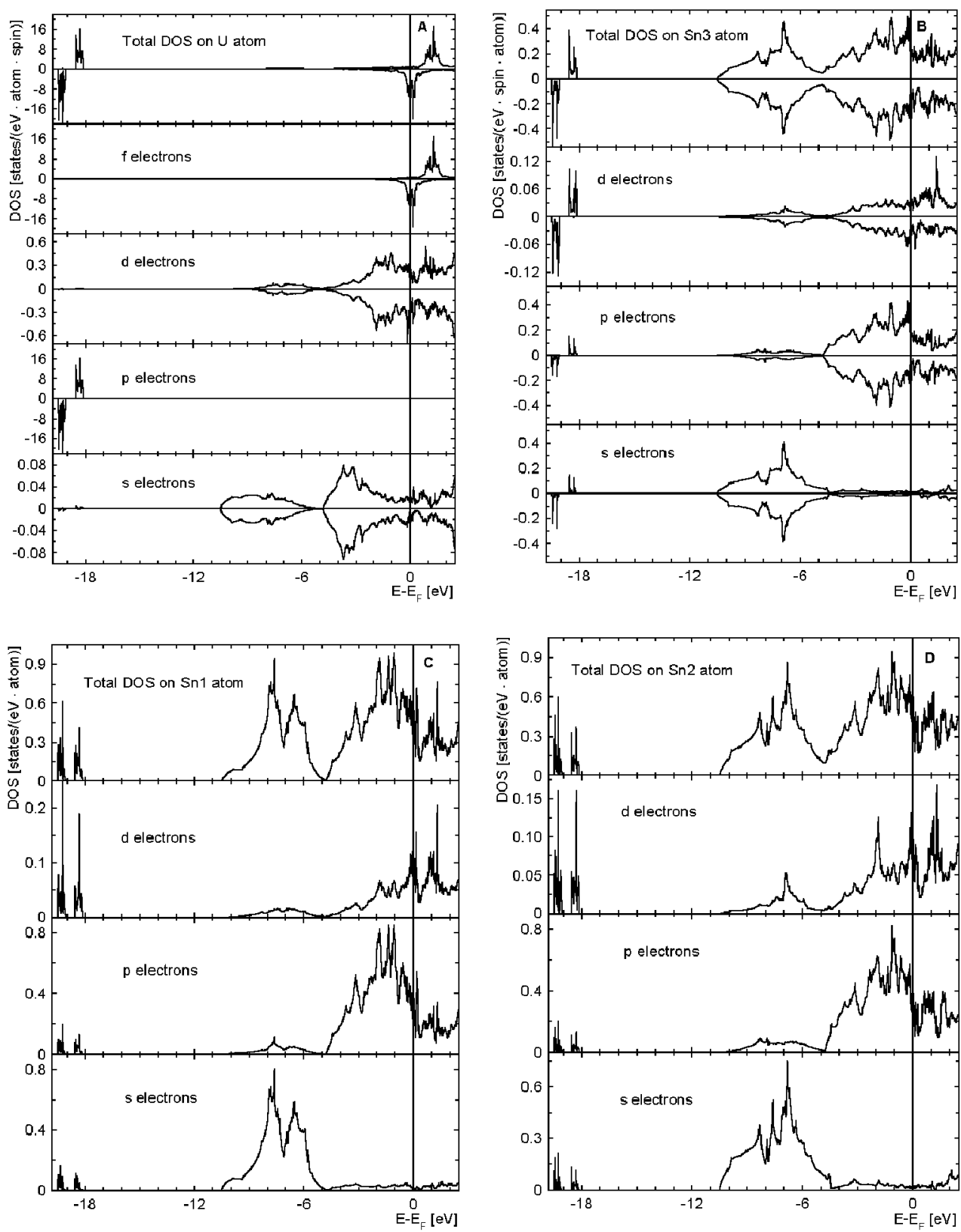

Fig. 3. The local and $l$-decomposed DOS plots for $\mathrm{USn}_{2}$ compound: (A) and (B) for U and Sn 3 atoms, respectively, presenting results for two spin directions; (C) and (D) for Sn1 and Sn2 atoms, respectively, presenting results for one spin direction (no magnetic moment on these atoms). 
TABLE II

Number of states for $\mathrm{USn}_{2}$ compound (per atom or unit cell and spin).

\begin{tabular}{c|c|c|c|c|c|c}
\hline \hline Atom (site) & Spin & $s$ & $p$ & $d$ & $f$ & Total \\
\hline $\mathrm{U}(4 \mathrm{j})$ & $\uparrow$ & 0.249 & 3.037 & 0.989 & 0.415 & 4.690 \\
& $\downarrow$ & 0.268 & 3.041 & 1.153 & 2.794 & 7.256 \\
$\mathrm{Sn} 1(4 \mathrm{i})$ & $\uparrow$ or $\downarrow$ & 0.785 & 1.062 & 0.120 & 0.046 & 2.013 \\
$\mathrm{Sn} 2(2 \mathrm{a})$ & $\uparrow$ or $\downarrow$ & 0.787 & 1.037 & 0.151 & 0.059 & 2.034 \\
$\mathrm{Sn} 3(2 \mathrm{c})$ & $\uparrow$ & 0.784 & 1.028 & 0.132 & 0.051 & 1.995 \\
& $\downarrow$ & 0.778 & 0.999 & 0.156 & 0.061 & 1.994 \\
Total & $\uparrow$ & 7.278 & 20.526 & 5.000 & 2.064 & 34.868 \\
(per cell) & $\downarrow$ & 7.342 & 20.484 & 5.706 & 11.600 & 45.132
\end{tabular}

TABLE III

Density of states at the Fermi level for $\mathrm{USn}_{2}$ compound (per atom or f.u. and spin).

\begin{tabular}{c|c|c|c|c|c|c}
\hline \hline Atom (site) & Spin & $s$ & $p$ & $d$ & $f$ & Total \\
\hline $\mathrm{U}(4 \mathrm{j})$ & $\uparrow$ & 0.020 & 0.014 & 0.238 & 0.402 & 0.674 \\
& $\downarrow$ & 0.021 & 0.024 & 0.288 & 9.867 & 10.200 \\
$\mathrm{Sn} 1(4 \mathrm{i})$ & $\uparrow$ or $\downarrow$ & 0.007 & 0.197 & 0.050 & 0.029 & 0.283 \\
$\mathrm{Sn} 2(2 \mathrm{a})$ & $\uparrow$ or $\downarrow$ & 0.009 & 0.223 & 0.053 & 0.053 & 0.338 \\
$\mathrm{Sn} 3(2 \mathrm{c})$ & $\uparrow$ & 0.011 & 0.155 & 0.035 & 0.009 & 0.210 \\
& $\downarrow$ & 0.003 & 0.157 & 0.037 & 0.051 & 0.248 \\
Total & $\uparrow$ & 0.037 & 0.400 & 0.332 & 0.462 & 1.231 \\
(per f.u.) & $\downarrow$ & 0.034 & 0.411 & 0.383 & 9.948 & 10.776
\end{tabular}

is provided by $\mathrm{U}(5 f)$ and $\mathrm{U}(6 d)$ electrons $\left(2.379\right.$ and $0.164 \mu_{\mathrm{B}} / \mathrm{U}$ atom, respectively). The magnetic moments in the unit cell presented in Fig. 1 are parallel. The antiferromagnetic situation is obtained expanding the cell along $c$ axis. A very small moment, antiparallel to the uranium one, is induced on $\mathrm{Sn} 3$ atom, $\approx 0.001 \mu_{\mathrm{B}} / \mathrm{Sn} 3$ atom. The resultant moment is an effect of opposite polarization of particular types of electrons. The contributions to the Sn3 total magnetic moment are the following: $0.006,0.029,-0.026$, and $-0.010 \mu_{\mathrm{B}}$ for $s, p, d$, and $f$ electrons, respectively. Comparing calculated spin magnetic moment of the $\mathrm{U}$ atom and the resultant experimental value $1.56 \mu_{\mathrm{B}} / \mathrm{U}$ atom [7] one can estimate orbital moment located on uranium atoms. The orbital moments on $\mathrm{U}$ atoms are antiparallel to the spin ones and their values are equal to about $4 \mu_{\mathrm{B}} / \mathrm{U}$ atom. The value is of the order of typical values obtained by fully relativistic codes reported for $\mathrm{UGe}_{2}\left(2.85-3.18\right.$ [16]), $\mathrm{UFe}_{5} \mathrm{Al}_{7}$ (2.44 [24]), or USb (3.42 [25]). 
For $\mathbf{U}$ and $\mathrm{Sn} 3$ atoms the spin projected DOS plots are presented in Figs. 1B, $2 \mathrm{~A}$, and $2 \mathrm{~B}$. In the case of $\mathrm{Sn} 3$ the differences in the spin projected DOS are visible only in the vicinity of the Fermi level. For Sn1 and Sn2 atoms (Figs. 3C, D) the spin projection is not necessary, the calculated moments are below $10^{-5} \mu_{\mathrm{B}} / \mathrm{Sn}$ atom, value beyond experimental resolution and within computational error.

\section{Conclusions}

The presented results of spin polarized band structure calculations for $\mathrm{USn}_{2}$ compound show that the magnetic moments are formed mainly by $\mathbf{U}(5 f)$ and $\mathrm{U}(6 d)$ electrons and order antiferromagnetically. The three types of Sn atoms may be treated as non-magnetic. The magnetic unit cell is composed of the crystallographic cell doubled along $c$ axis.

\section{Acknowledgment}

Work supported by the State Committee for Scientific Research grant No. 2 P03B 02322 and the Centre of Excellence for Magnetic and Molecular Materials for Future Electronics within the European Commission contract No. G5MA-CT-2002-04049. The calculations were performed at the Supercomputing and Networking Centre of Poznań (PCSS).

\section{References}

[1] D.A. Treick, J.H. Carter, A.I. Snow, B.R. Baldwin, A.S. Wilson, The Uranium-Tin System, US Atomic Energy Comm. M-3107 (1945).

[2] R.I. Sheldon, E.M. Foltyn, D.E. Peterson, Bull. Alloy Phase Diagrams 8, 347 (1987)

[3] C. Sari, F. Varenza, W. Muller, J. Less Common Met. 92, 301 (1983).

[4] A. Palenzona, P. Manfrinetti, J. Alloys Comp. 221, 157 (1995).

[5] P. Boulet, H. Noël, Solid State Commun. 107, 135 (1998).

[6] S.K. Dhar, Y. Kimura, M. Kouzaki, K. Sugiyama, R. Settai, Y. Onuki, T. Takeuchi, K. Kindo, P. Manfrinetti, A. Palenzona, Physica B 245, 210 (1998).

[7] P. Boulet, G. André, F. Bourée, H. Noël, J. Alloys Comp. 329, 47 (2001).

[8] A. Hasegawa, J. Magn. Magn. Mater. 52, 425 (1985).

[9] D.D. Koelling, M.R. Norman, A.J. Arko, J. Magn. Magn. Mater. 63-64, 638 (1987)

[10] M. Olšovec, M. Diviš, Phys. Status Solidi B 195, K13 (1996).

[11] A.L. Cornelius, A.J. Arko, J.L. Sarrao, J.D. Thompson, M.F. Hundley, C.H. Booth, N. Harrison, P.M. Oppeneer, Phys. Rev. B 59, 14473 (1999).

[12] A. Szajek, Phys. Status Solidi B 236, 552 (2003).

[13] A. Szajek, J. Magn. Magn. Mater. 272-276, e347 (2004). 
[14] H. Takahashi, N. Mori, Y. Onuki, S.W. Yun, Physica B 186-188, 772 (1993).

[15] S.S. Saxena, P. Agarwal, K. Ahilan, F.M. Grosche, R.K.W. Haselwimmer, M.J. Steiner, E. Pugh, I.R. Walker, S.R. Julian, P. Monthoux, G.G. Lonzarich, A. Huxley, I. Sheiken, D. Braithwaite, J. Flouquet, Nature 406, 587 (2000).

[16] A.B. Shick, W.E. Pickett, Phys. Rev. B 86, 300 (2001).

[17] O.K. Andersen, O. Jepsen, M. Šob, in: Electronic Structure and Its Applications, Ed. M.S. Yussouff, Springer, Berlin 1987, p. 2.

[18] G. Krier, O. Jepsen, A. Burkhardt, O.K. Andersen, The TB-LMTO-ASA Program, source code version 4.7, available upon request from the program authors.

[19] B.I. Min, Y.-R. Jang, J. Phys., Condens. Matter 3, 5131 (1991).

[20] U. von Barth, L. Hedin, J. Phys. C 5, 1629 (1972).

[21] P. Blöchl, O. Jepsen, O.K. Andersen, Phys. Rev. B 49, 16223 (1994).

[22] A. Szajek, Cryst. Res. Technol. 36, 1105 (2001).

[23] G. Chełkowska, J.A. Morkowski, A. Szajek, R. Troć, J. Phys., Condens. Matter 14, 3199 (2002).

[24] C. Cardoso, L.M. Sandratskii, T. Gasche, M. Godinho, Phys. Rev. B 65, 094413 (2002).

[25] H. Yamagami, Phys. Rev. B 61, 6246 (2000). 